\title{
Mental Health Literacy of Depression and Postnatal Depression: A Community Sample
}

\author{
Einar B. Thorsteinsson, Natasha M. Loi, Anna L. Moulynox \\ Psychology, School of Behavioural, Cognitive and Social Sciences, University of New England, Armidale, \\ Australia \\ Email: ethorste@une.edu.au, einarbt@gmail.com
}

Received 22 May 2014; revised 28 June 2014; accepted 20 July 2014

Copyright (C) 2014 by authors and Scientific Research Publishing Inc.

This work is licensed under the Creative Commons Attribution International License (CC BY).

http://creativecommons.org/licenses/by/4.0/

c) (i) Open Access

\begin{abstract}
Purpose: The present experimental study examined mental health literacy of postnatal depression (PND) in an Australian community sample. Method: Five hundred and twenty Australians (427 females) participated in an experimental study. Participants were randomly presented with one of three vignettes describing a "depressive" episode and then completed an online questionnaire examining mental health literacy and the capacity to recognise PND and its risk factors as well as the ability to source appropriate information. Results: Generally, the ability to recognise PND and the overall mental health literacy of the sample were high. Causation, however, was often misattributed to biological or environmental/social factors. PND recognition was found to be significantly affected by perception of the attachment relationship presented in two of the vignettes. Conclusions: Perception of attachment impacts PND recognition and this may negatively impact help-seeking behaviours.
\end{abstract}

\section{Keywords}

Postnatal Depression, Depression, Attachment, Causal Attribution, Risk Factors

\section{Introduction}

Mental illness has been identified as the leading cause of disability in Australia and costs an estimated \$20 billion annually in lost productivity (Australian Bureau of Statistics, 2010). Furthermore, mental illness disrupts an individual's ability to relate to their family, friends, colleagues, and broader community, leading to significant distress and disability. In 2007, 45\% of Australians, or 7.3 million individuals, reported that they had experienced a mental illness during their lives (Australian Bureau of Statistics, 2010). The National Health and Wellbeing Survey (Australian Bureau of Statistics, 2007) reported that of the individuals who reported expe-

How to cite this paper: Thorsteinsson, E.B., Loi, N.M., \& Moulynox, A.L. (2014). Mental Health Literacy of Depression and Postnatal Depression: A Community Sample. Open Journal of Depression, 3, 101-111.

http://dx.doi.org/10.4236/ojd.2014.33014 
riencing a mental illness in the previous 12 months, 65\% had not accessed any services. When asked why, they often reported that they had no need for any type of assistance. Therefore, assuming that there are services available and individuals are aware of them, the question is why they feel they do not need assistance. The answer may lie in mental health literacy.

In the present study, mental health literacy was examined as it relates to postnatal depression (PND), a common depressive disorder affecting a significant proportion of Australian mothers. Mental health literacy for PND has not received much attention or been widely measured in Australia, and with a vast array of misconceptions surrounding its diagnosis (e.g., such as a lack of distinction between PND, "baby blues" and postpartum psychosis). This study examined individuals' abilities to recognise PND, identify risk factors and causes, and determine what sources of information and help they would recommend to someone experiencing PND.

\subsection{Mental Health Literacy}

Mental health literacy is defined as "knowledge and beliefs about mental disorders which aid their recognition, management or prevention" (Jorm et al., 1997: p. 182). This includes the ability to recognise particular disorders, knowing how to seek mental health information, knowledge of risk factors and causes, knowledge about available self-treatments and professional help, and attitudes that promote appropriate help-seeking (Jorm et al., 1997). According to Jorm et al.'s definition, an individual with good mental health literacy would be able to identify mental illness in themselves or others and then seek or recommend appropriate sources of help. However, research into mental health literacy in Australia has shown it to be generally poor (Goldney, Fisher, \& Wilson, 2001; Goldney, Fisher, Wilson, \& Cheok, 2000). High levels of mental health literacy will increase the likelihood that these mental illnesses will be recognised and this will increase the possibility of appropriate intervention and meaningful social support (Jorm \& Kelly, 2007).

In 2000, the Australian government sought to redress the knowledge deficit with respect to depression by investing in primary health care and increasing the availability of services (Whiteford, Buckingham, \& Manderscheid, 2002). To give voice to the strategy of education and communication about depression, a health promotion agency, beyondblue, was developed to raise community awareness and reduce stigma. Although mental health literacy for depression may have improved following beyondblue's inception, similar improvement cannot be expected for other mood disorders such as postnatal depression. Mental health literacy for PND, nonpsychotic depression diagnosed either during pregnancy or the first four weeks postpartum (American Psychiatric Association, 2013), has not been systematically assessed in the Australian public. Thus the aim of the current study is to investigate mental health literacy relating to PND in an Australian community sample.

\subsection{Postnatal Depression}

PND affects 16\% of Australian mothers (Buist et al., 2008). The Diagnostic and Statistical Manual of Mental Disorders-5 (American Psychiatric Association, 2013) lists peripartum onset as a specifier for a major depressive episode. Thus PND is based on the same diagnostic criteria as a major depressive episode, but with the unique requirement that the symptoms (e.g., depressed mood, loss of interest or pleasure, significant weight gain or loss, guilt) occur during pregnancy or in the postnatal period.

In the absence of a mental health literacy survey, researchers have examined the content of popular press articles to gauge public understanding of PND. Schanie, Pinto-Foltz, and Logsdon (2008), for example, found that $36 \%$ of the articles identified fear of harming the baby or fear of the baby's death as a symptom, and $34 \%$ identified lack of interest in the baby as a symptom of PND rather than an effect. Furthermore, published symptom lists are often incomplete and inaccurate with little distinction made between "baby blues," PND, and postpartum psychosis (Martinez, Johnston-Robledo, Ulsh, \& Chrisler, 2001).

Fears of harming the baby and poor attachment with the baby are often described in anecdotal reports on PND (Fernandez, 1992). While research confirms that fear of harming the infant is common in PND (Fairbrother \& Woody, 2008) this is highly uncommon behaviour in PND sufferers (Donahue Jennings, Ross, Popper, \& Elmore, 1999). With regards to PND and attachment, the research findings are clear that long term, PND interferes with the attachment between mother and child. Mothers experiencing PND tend to be less responsive and more inconsistent in their care of the infant (Figueiredo, Costa, Pacheco, \& Pais, 2009; Hendrick, 2003; Moehler, Brunner, Wiebel, Reck, \& Resch, 2006). However, the community perception seems to be that poor attachment with the infant is considered to be a symptom of PND rather than an effect (beyondblue, 2009). Thus the current 
research will examine if perception of attachment between mother and child will affect perception of diagnosis.

Recognition of PND presents some unique difficulties. Many women assume that symptoms are a normal part of parenthood and the result of fatigue, relationship strain, and personal weakness rather than depression and these perceptions are often supported by family members (Letourneau et al., 2007). Research findings such as these emphasise the vital nature of increasing community mental health literacy for PND.

The Australian government has already introduced some initiatives to improve screening and treatment for PND. In 2009 the National Perinatal Depression Initiative was introduced and all pregnant women and new mothers are routinely screened for depression. This will increase the number of cases identified, but it may depend on an individual's mental health literacy as to whether they will then engage with treatment (Highet, Gemmill, \& Milgrom, 2011).

It is important for the causes of PND to be recognised as the misattribution of cause can also hinder appropriate help seeking in those affected. Research shows that instead of a definitive cause for PND, there are recognised risk factors. The precise combination of risk factors is unique to each woman, these include: a history of depression or anxiety; stressful life events; lack of social support; poor marital relationship; neuroticism; low self-esteem; difficulties with child care; difficult infant temperament; baby blues; obstetric complications; low socioeconomic status; single marital status; unplanned or unwanted pregnancy; preterm birth; and multiple births (Beck, 2001; Milgrom et al., 2008; Robertson, Grace, Wallington, \& Stewart, 2004).

The use of psychological therapies such as cognitive behavioural and group therapies as the first-line treatment for PND has been advocated (Austin, Highet, \& Guidelines Expert Advisory Committee, 2011) as a result of the evidence found for their effectiveness (e.g., Bledsoe \& Grote, 2006; Cuijpers, Brännmark, \& van Straten, 2008). Despite this, many GPs prescribe antidepressants to women with PND (Turner, Sharp, Folkes, \& ChewGraham, 2008). Breastfeeding women may be reluctant to take medication, however, and their beliefs about side effects, dependence, and stigma associated with taking antidepressants may result in poor treatment adherence (Turner et al., 2008). While PND is a treatable condition and both pharmacological and psychological interventions can be of help, it would appear that treatment for PND needs to be more comprehensive. Research by Forman et al. (2007) reveals that even if PND is treated, this alone is not enough to protect the infant from the effects of having a depressed parent. Depressed mothers are often quite high functioning, however, they are significantly less responsive to their infants, experience more parenting stress and rate their infant's temperaments as more negative than non-depressed mothers. While the mothers in Forman et al.'s study were effectively treated for their PND, this treatment had no discernible effect on maternal responsiveness. While treating the mother's PND is important, it will not repair the attachment between mother and child and so additional help is required to re-establish the relationship. This is an additional reason behind the need for early symptom recognition and regular screening so that assistance can be provided before maternal attachment is irreparably damaged.

In summary, PND is a common depressive disorder commonly diagnosed up to 12 months following birth, and while having no exact cause, there are many well-researched risk factors. Many misconceptions about the disorder exist suggesting that mental health literacy relating to PND is likely to be low. This is of concern as research has shown that low mental health literacy in regards to PND is one of the most significant barriers to help-seeking behaviour and contributes directly to delayed help seeking (Thompson, Issakidis, \& Hunt, 2008). The occurrence of PND not only affects the mother, it can also have a profound effect on the parent-child attachment. Mothers with PND are less likely to be consistent in their responses to their baby and are more likely to be avoidant which can result in insecure attachment in the infant (Murray \& Cooper, 1999). Thus the more promptly an individual can seek help, the less damage is done to the parent-child attachment.

\subsection{The Current Study}

The purpose of the current study is to examine mental health literacy relating to PND by assessing ability to recognise PND from depression, to identify risk factors/causes, and to identify appropriate sources of help and information. Findings will enable the identification of common misconceptions of PND in the Australian public. In accordance with the literature reviewed, it was hypothesised that: 1) when presented with a vignette either describing a depressed individual or a depressed individual in the postnatal period, participants would be able to make a correct diagnosis of depression or PND, respectively; 2) when presented with a vignette describing an individual with PND, participants' diagnoses would be affected by the description of attachment to the infant, with poor attachment leading to an increased diagnosis of PND; 3) participants' suggested sources of a) infor- 
mation; b) help; c) treatment; and d) suggested causative factors of PND would differ according to diagnosis; and 4) participants would expect duration of illness to decrease if the individual received treatment.

\section{Method}

\subsection{Participants}

A total of 520 individuals participated in the present study. Of the total, 20 were excluded from the final data analysis due to inadequate completion of the questionnaire, leaving a final number of 500 participants. A power analysis suggested that a sample of 180 participants (60 per vignette), assuming alpha set at .05 , and a medium effect size would give adequate power $(>.80)$.

Participants' ages ranged from 18 to 75 years $(M=33.73, S D=9.55)$, with the majority being female $(85.4 \%$, $n=427)$, parents $(73.2 \%, n=366)$, and holding a university degree $(54.6 \%, n=273)$. For respondents who were parents, the average age at birth of their first child was 26.94 years $(S D=5.12$; range from 16 to 42 years, $n=$ $364)$, and the modal number of children was two $(M=2.18, S D=1.07$; range from 1 to 8$)$.

Recruitment was via invitations on notice boards at a local community health centre and university. Online invitations were issued via the research forum on www.bubhub.com.au, direct email, and Facebook.

\subsection{Materials}

Three vignettes were developed based on the work by Jorm in 1997 and personal communication with Jorm (A. Jorm, personal communication, October 25, 2010). The first vignette (Depression-control) meets the diagnostic requirements for Major Depressive Episode as per the DSM-5, the second vignette (PND-poor attachment) meets the diagnostic requirements of Major Depressive Episode with Peripartum Onset Specifier and makes reference to attachment/bonding problems, and the third vignette (PND-good attachment) also meets the diagnostic requirements of Major Depressive Episode with Peripartum Onset Specifier and makes reference to positive attachment/bonding between mother and child.

The initial questionnaire consisted of three sections. The first contained 12 items designed to investigate participants' responses to the presented vignette. These items were based (with permission) on the work of Jorm and colleagues (A. Jorm, personal communication, October 25, 2010) and asked participants questions relating to what they thought was wrong with the character in the vignette, where could she find help, and how long the problem would last after receiving treatment. This was followed by a section of 16 demographic questions. These also included questions pertaining to training in, experiences of, and information sourcing for PND and mental health in general (e.g., "Have you or someone you know experienced postnatal depression?”, “Are you aware of the organisation beyondblue?”). The final section contained 43 true/false/not sure questions about PND; these questions were based on information from beyondblue, the Post and Antenatal Depression Association (PANDA), and the DSM-5 symptom list for depression (e.g., "PND is different from the 'baby blues'”, "Antidepressants can be part of a good treatment plan for PND”). These were intended to test participants' knowledge of PND and some of the myths surrounding PND.

The questionnaire was piloted with six individuals resulting in a change of question presentation order, changes to wording and the removal of some items. The final version of the survey commenced with a participant information page followed by 13 questions in section one, 16 demographic questions in section two, and 36 true/false questions in section three. At the conclusion of the questionnaire participants were provided with phone numbers for telephone counselling, a link to the PANDA website and comprehensive information about PND (reproduced with permission) from the PANDA factsheets (A. Christofidis, personal communication, March 28, 2011).

\subsection{Procedure}

After reading the online information sheet and consenting to participate in the study each participant was randomly presented one of three vignettes. Random allocation to vignette was conducted employing in the Qualtrics online survey interface. Following this participants were then presented with the questionnaire.

\subsection{Ethics}

This study was conducted with full human research ethics committee approval by the University of New Eng- 
land, Australia, Human Research Ethics Committee. All interactions with participants were online. All participants were 18 years of age or older. All participants viewed an information sheet online before commencing the questionnaire; the information sheet outlined the voluntary and anonymous nature of the survey and stated that the participant could withdraw from the study at any time. Participants consented to participate in the study by proceeding past the information screen to the questionnaires. This consent procedure fulfilled all requirements of the University of New England, Australia, Human Research Ethics Committee.

\subsection{Statistical Analyses and Interpretation}

SPSS version 19 was used for statistical analyses. The effect size reported, $d$, is the standard deviation unit Hedges' $g$ (unbiased) and similar to Cohen's $d$ (Borenstein, Hedges, Higgins, \& Rothstein, 2009). The convention of small (0 to 0.2), medium (0.2 to 0.8), and large ( $>0.8$ ) was used (Cohen, 1988). Corresponding Cramer's $V$ and $\phi$ effect size values are .00 to .10 for small, $>.10$ and $<.37$ for medium, and .37 and higher for large.

\section{Results}

The raw data were first examined for accuracy and missing values. Of the 500 participants, 169 were exposed to the PND-poor attachment vignette, 169 the vignette of PND-good attachment, and 162 the depression-control vignette.

As outlined in Table 1, three quarters of respondents reported that they knew someone who had experienced PND, and a third indicated that it had been themselves or their partner. Most respondents indicated that they knew someone who had experienced a mental illness other than PND; friends, family members, and self/partner were the most reported.

All vignettes described a depressed individual and two described a depressed individual in the postnatal period. There was a strong association between vignette presented and diagnosis given, $\chi^{2}(8, N=496)=265.83, p$ $<.001$ (two-tailed), large effect size Cramer's $V=.52$. As illustrated in Figure 1, participants were more likely to make a diagnosis of depression when presented with the depression vignette (74\%) and PND when presented with the PND vignette (77.5\%) than any other diagnosis. There was a small but significant association between diagnosis given and attachment statement, $\chi^{2}(1, N=262)=3.89, p=.048$ (two-tailed), medium effect size $\phi=$ -.12. As illustrated in Figure 2, participants were more likely to make a diagnosis of PND when the vignette referred to poor attachment.

As participants were able to select multiple responses, this data did not satisfy the assumption of independence for hypothesis 3, thus chi-square analysis was not used. Rather, descriptive statistics (i.e., frequencies and percentages) were used to examine the data (see Table 2). Participants' suggested sources of information were very similar for both PND and depression diagnosis (irrespective of vignette) with the exception of community health nurse which was suggested more for PND. Suggested sources of help were again very similar with the exception of community health nurse which was suggested more for PND. Recommended forms of treatment differed more between the groups. Family support, relaxation/time to self, support groups, and sleep were more frequently recommended for depression. Causative factors were the most disparate. Hormonal changes, being unprepared and uninformed about parenthood, single parent status, having a difficult baby, obstetric factors, and lack of sleep were all more frequently cited as contributing to PND, whereas day-to-day problems, traumatic events, virus or infection, being a nervous person, genetic tendency, low self-esteem, marital problems, and financial problems were more frequently cited as contributing to depression.

Finally, for hypothesis 4 participants were asked to estimate time of recovery (weeks) assuming no treatment ( $M=26.99, S D=16.79)$ or assuming treatment they recommended $(M=12.35, S D=11.03)$. There was a statistically significant difference between estimated recovery time with and without treatment. On average, respondents estimated recovery time to be 14.6 weeks shorter with their recommended treatment than without, $95 \%$ CI [12.8, 16.5], $t(259)=15.62, p<.001$ (two-tailed), large effect size $d=1.05$.

\section{Discussion}

The first hypothesis was supported given high percentage recognition of depression, $74 \%$, which is similar to the 67\% reported by Highet, Luscombe, Davenport, Burns and Hickie (2006). The results of this study suggest that the increase in depression recognition has extended to depression in the postnatal period. The current study 
Table 1. Experience with Postnatal Depression (PND) and other mental health issues.

\begin{tabular}{ccc}
\hline Variable & $\mathrm{n}$ & $\%$ \\
PND experience & & 73.2 \\
Yes & 366 & 26.8 \\
No & 134 & 18.0 \\
Relationship to person with PND & & 22.2 \\
Close friend & 90 & 7.4 \\
Friend & 111 & 34.6 \\
Acquaintance & 37 & 18.0 \\
Self or partner & 173 & 5.4 \\
Family member & 90 & 3.4 \\
Parent & 27 & 94.6 \\
Other & 17 & 5.4 \\
Mental Illness experience (other than PND) & & 29.6 \\
Yes & 473 \\
No & 27 & 29.6 \\
Close friend & & 16.2 \\
Friend & 148 & 39.8 \\
Acquaintance & 148 & 45.6 \\
Self or partner & 81 & 18.4 \\
Family member & 199 & 4.6 \\
Parent & 228 & \\
\hline
\end{tabular}

Note: Respondents were permitted to choose multiple options, thus percentages may add up to more than 100.

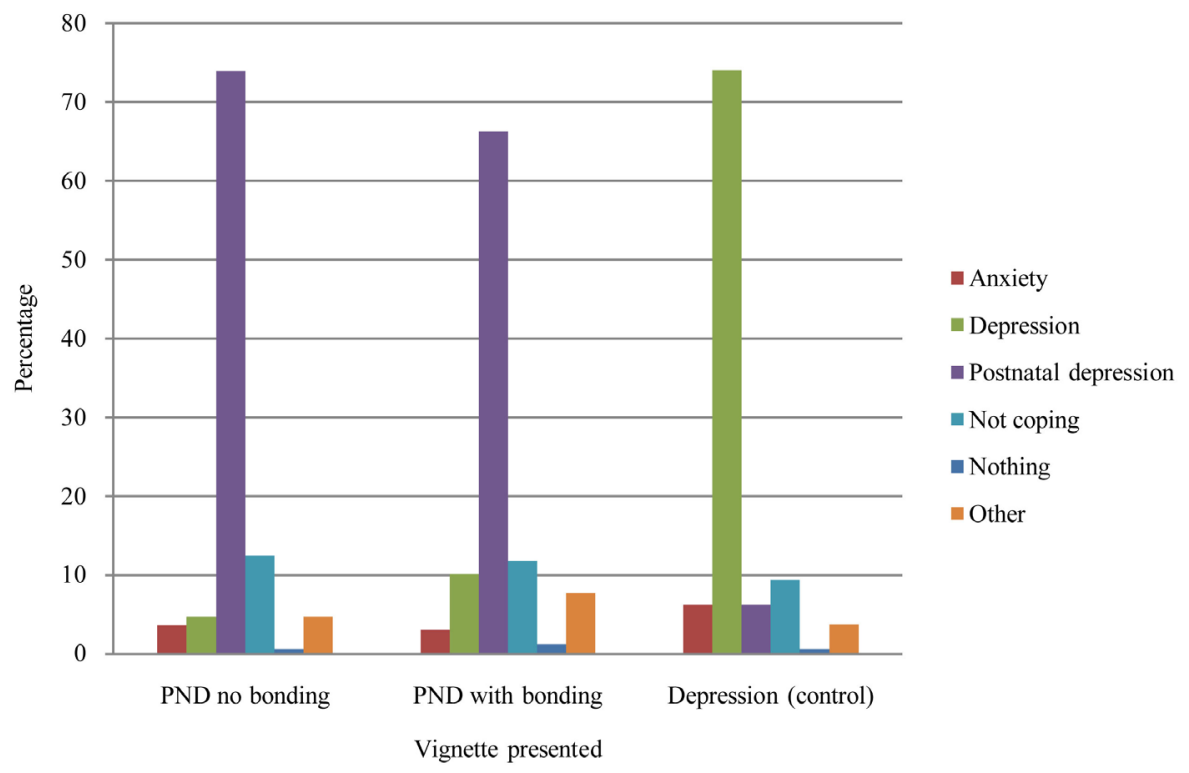

Figure 1. Clustered bar chart illustrating the diagnosis given according to vignette presented.

showed a PND recognition rate of $77.5 \%$ whereas Highet et al. (2011) revealed a recognition rate of $43.6 \%$. This difference may be due to the different composition of the samples. The current study had more female respondents compared with Highet et al. (2011), 85\% versus 65\%, and the age distribution was markedly different, with many more respondents in the 18 to 34 age group and less in the over 55 age group in the present study. 
Table 2. Recommended sources of information, help and treatment and suggested causative factors by diagnosis in descending order by PND.

\begin{tabular}{|c|c|c|c|c|}
\hline \multirow[b]{2}{*}{ Source } & \multicolumn{2}{|c|}{ PND } & \multicolumn{2}{|c|}{ Depression } \\
\hline & $\mathrm{n}$ & $\%$ & $\mathrm{n}$ & $\%$ \\
\hline \multicolumn{5}{|l|}{ Information Source } \\
\hline $\mathrm{GP}$ & 237 & 96 & 141 & 97 \\
\hline Community health nurse & 213 & 89 & 107 & 74 \\
\hline Telephone counselling service & 208 & 84 & 125 & 86 \\
\hline Psychologist & 197 & 80 & 113 & 78 \\
\hline Internet & 196 & 79 & 112 & 77 \\
\hline Family & 165 & 67 & 94 & 65 \\
\hline Friends & 156 & 63 & 93 & 64 \\
\hline Psychiatrist & 132 & 53 & 77 & 53 \\
\hline Pharmacist & 84 & 34 & 44 & 30 \\
\hline Other & 17 & 7 & 8 & 6 \\
\hline \multicolumn{5}{|l|}{ Help Source } \\
\hline GP & 236 & 96 & 138 & 95 \\
\hline Community health nurse & 213 & 75 & 128 & 54 \\
\hline Psychologist & 198 & 80 & 125 & 86 \\
\hline Minister/priest & 184 & 27 & 78 & 34 \\
\hline Friends & 176 & 68 & 112 & 69 \\
\hline Psychiatrist & 173 & 53 & 95 & 58 \\
\hline Counsellor & 168 & 86 & 100 & 88 \\
\hline Telephone counselling service & 149 & 71 & 79 & 77 \\
\hline Family & 134 & 70 & 70 & 66 \\
\hline Internet & 130 & 54 & 84 & 48 \\
\hline Naturopath/herbalist & 66 & 24 & 49 & 35 \\
\hline Social worker & 58 & 60 & 51 & 55 \\
\hline Pharmacist & 45 & 18 & 16 & 11 \\
\hline Other & 11 & 5 & 6 & 4 \\
\hline \multicolumn{5}{|l|}{ Recommended Treatment } \\
\hline Family support & 218 & 88 & 108 & 75 \\
\hline Support group & 209 & 85 & 93 & 64 \\
\hline Counselling/psychotherapy & 201 & 81 & 128 & 88 \\
\hline Relaxation/time to self & 188 & 76 & 96 & 66 \\
\hline Sleep & 183 & 74 & 83 & 57 \\
\hline Exercise & 182 & 74 & 118 & 81 \\
\hline Antidepressant medication & 137 & 56 & 86 & 59 \\
\hline Improved diet & 126 & 51 & 91 & 63 \\
\hline Cognitive therapy & 83 & 34 & 55 & 38 \\
\hline Childcare & 62 & 25 & 5 & 3 \\
\hline Herbal medication & 29 & 12 & 34 & 23 \\
\hline Other & 9 & 4 & 6 & 4 \\
\hline Electroconvulsive therapy & 3 & 1 & 0 & 0 \\
\hline Alcohol or illicit drugs & 2 & 1 & 1 & 1 \\
\hline \multicolumn{5}{|l|}{ Causative Factors } \\
\hline Hormonal changes & 224 & 91 & 115 & 79 \\
\hline Lack of sleep & 217 & 88 & 113 & 78 \\
\hline Lack of social support & 186 & 75 & 122 & 84 \\
\hline
\end{tabular}




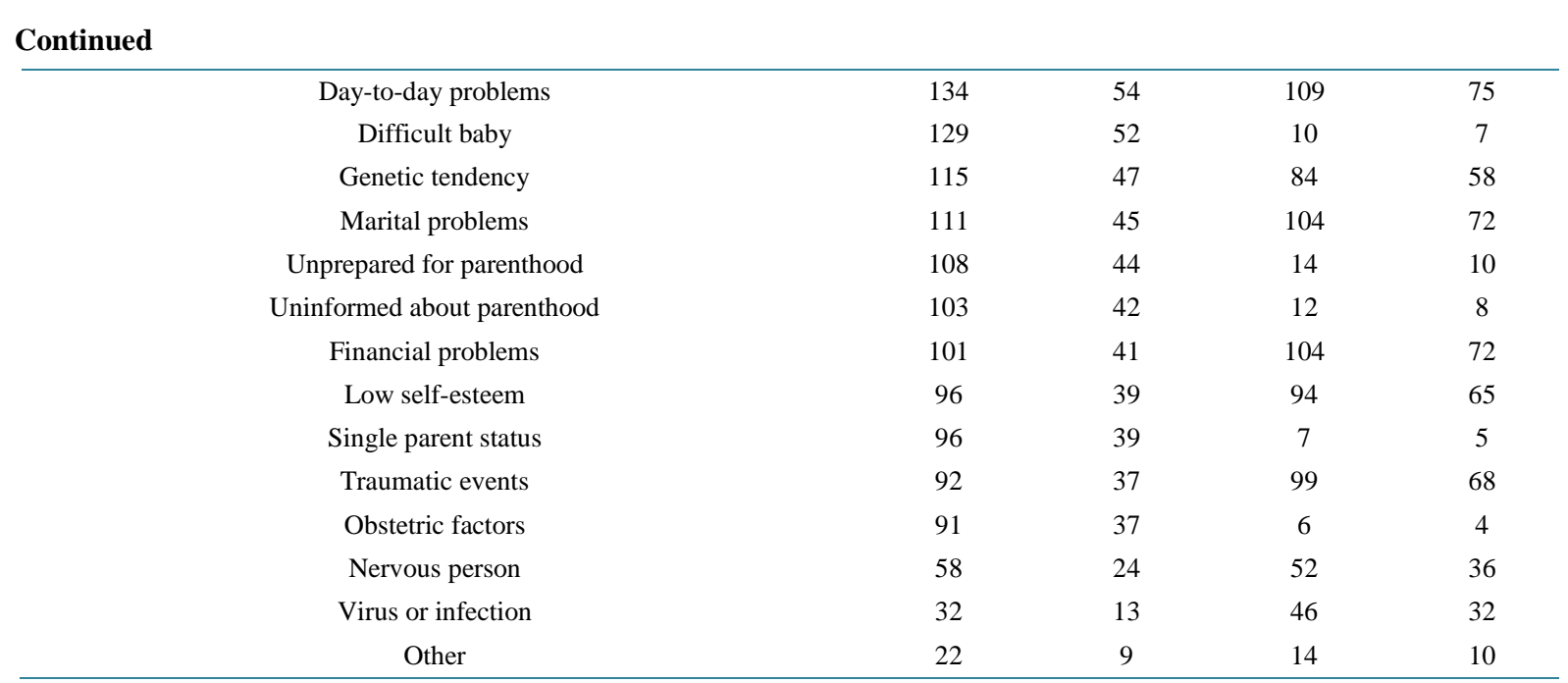

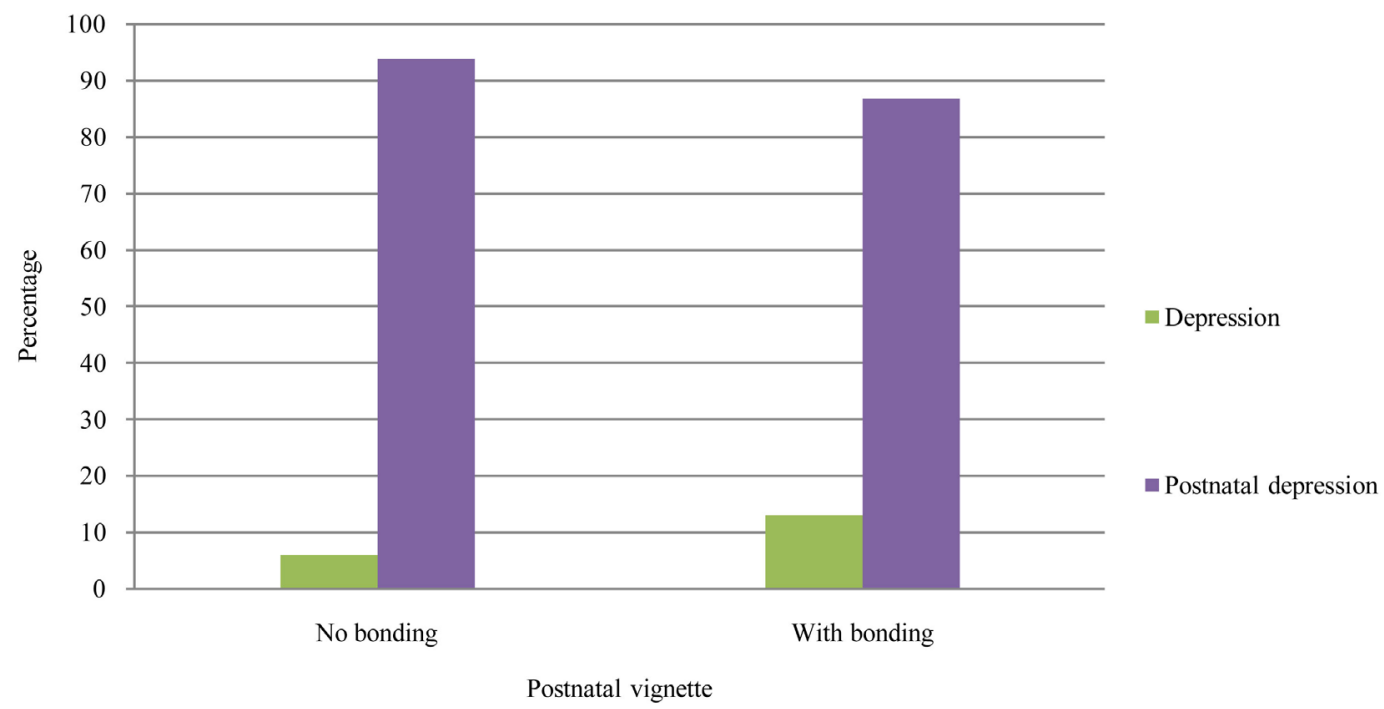

Figure 2. Clustered bar chart illustrating the diagnosis given for the postnatal depression vignettes.

Given the differences in these samples, the disparity between PND recognition rates in the two studies is consistent with previous findings on mental health literacy. Young female adults are the group most likely to correctly identify depression with the elderly being the least likely (Cotton, Wright, Harris, Jorm, \& McGorry, 2006; Farrer, Leach, Griffiths, Christensen, \& Jorm, 2008; Fisher \& Goldney, 2003). Despite the disparity, the results from both Highet et al. (2011) and the current study suggest that recognition of PND in the community is at a high level. Highet et al. (2011) examined the beliefs of Australians about incidence rates of depression in the perinatal period. They found that this overestimation may inadvertently reduce stigma about depression in new mothers.

The second hypothesis stating that when presented with a vignette describing an individual with PND, participants' diagnoses would be affected by the description of attachment with the infant, was supported. When the vignette referred to poor attachment, participants were more likely (medium effect size) to make a diagnosis of PND. This is a novel finding with the implication being that this misconception may be hindering recognition of PND and presenting an additional barrier to help seeking. When asked about the "facts" of PND, many participants showed good knowledge, but if recognition is confounded by perception of attachment, then help may not be sought or recommended. Anecdotally, when individuals are questioned about PND they often list poor attachment or failure to bond as a symptom essential to diagnosis rather than as a cause stemming from ongoing lack of interaction with the infant. In the current study only $13 \%$ of respondents were unsure or thought that 
failure to bond was a diagnostic criterion. This suggests that although the facts of PND are understood, when faced with a situation such as the vignette, current thinking appears to lead individuals to the conclusion that if a mother loves her child then it must be something other than PND that is wrong. This finding merits further investigation.

The results supported the third hypothesis for treatment and causative factors but not for information and help. This suggests that participants' sources of information and help are consistent for varying mental illnesses, but there is more confusion surrounding causation and thus treatment for PND. PND as compared with depression was more likely to be seen as the result of lack of preparation/information, biological issues, and environmental factors such as being a single parent or having a difficult baby. Infant temperament, obstetric complications, and single parent status are recognised as being common risk factors, but the frequently cited hormonal factors are not (Beck, 2001; Milgrom et al., 2008; Robertson et al., 2004).

There were many commonly suggested treatments. The recommendations of therapy and antidepressants are likely to be part of the "beyondblue effect", while recommendations of laypersons' networks for PND is likely to be a reflection of how PND is understood. Recommendations such as these may suggest that PND is seen to have more of a social or environmental provenance rather than being something uncontrollable by nature (Angermeyer, Matschinger, \& Riedel-Heller, 1999). Sources of information and help were very similar for PND and depression with the exception of community health nurses who were recommended more highly for PND than for depression as both a source of information and help. This is positive in light of the additional funding and training that was provided for these services (Department of Health and Ageing, 2009).

Finally, the fourth hypothesis was supported, with participants, on average, expecting recovery time to be almost 15 weeks shorter if their recommended treatment was implemented. This result is positive, especially when combined with the majority (91\%) of participants nominating "Support from family and friends are important but someone with PND also needs professional help.” This suggests that most participants see treatment as helpful for PND. As the most recommended treatments included counselling/psychotherapy, antidepressants, and support groups, this suggests that the awareness of treatment options for PND in the sample was good.

\subsection{Limitations}

Employing an Internet-based survey to collect data may have influenced sampling. The participants were predominantly non-Indigenous, female, well-educated, and parents, all of which may need to be taken into account when viewing the results of this sample in relation to the broader community. When asking about experience of mental illness, the questionnaire asked whether this related to "yourself or partner". Separating this question into two questions would have allowed a more detailed evaluation of the data. Finally, it is possible that the title of the questionnaire (Perceptions of Postnatal Depression) might have acted as a prompt thus inflating the correct percentage identified. However, any deception to try and hide this was ruled out a) for ethical reasons; b) the vignettes had to include real life information for identification, and c) real life provides prompts to family and friends such as the birth of a baby.

\subsection{Conclusion}

While PND is a common depressive disorder, misconception about the condition still remains, especially with respect to causation, though overall mental health literacy in the present sample was high. Prevailing misconceptions may impact help-seeking behaviours and thus impact future mental health.

\section{Conflict of Interest}

The authors declare that there is no conflict of interests regarding the publication of this article.

\section{References}

American Psychiatric Association (2013). Diagnostic and Statistical Manual of Mental Disorders (5th ed.). Arlington, VA: American Psychiatric Association.

Angermeyer, M. C., Matschinger, H., \& Riedel-Heller, S. G. (1999). Whom to Ask for Help in Case of a Mental Disorder? Preferences of the Lay Public. Social Psychiatry and Psychiatric Epidemiology, 34, 202-210.

Austin, M. P., Highet, N., \& Guidelines Expert Advisory Committee (2011). Clinical Practice Guidelines for Depression and 
Related Disorders_-Anxiety, Bipolar Disorder and Puerperal Psychosis—In the Perinatal Period. A Guideline for Primary Care Health Professionals. beyondblue, The National Depression Initiative, Melbourne.

Australian Bureau of Statistics (2007). National Survey of Mental Health and Wellbeing: Summary of Results 2007. Cat. No. 4326.0. http://www.abs.gov.au/ausstats/abs@.nsf/mf/4326.0

Australian Bureau of Statistics (2010). Year Book Australia 2009-10. Cat. No. 1301.0. http://www.abs.gov.au/ausstats/abs@.nsf/Previousproducts/1301.0Main\%20Features12009-10?opendocument\&tabname= Summary\&prodno=1301.0\&issue=2009-10\&num=\&view=

Beck, C. T. (2001). Predictors of Postnatal Depression: An Update. Nursing Research, 50, 275-285.

beyondblue (2009). What Australians Know about Perinatal Depression and Anxiety. beyondblue Perinatal Monitor 2009. https://www.bspg.com.au/dam/bsg/product?client=BEYONDBLUE\&prodid=BL/0788\&type=file

Bledsoe, S. E., \& Grote, N. K. (2006). Treating Depression during Pregnancy and the Postpartum: A Preliminary MetaAnalysis. Research on Social Work Practice, 16, 109-120. http://dx.doi.org/10.1177/1049731505282202

Borenstein, M., Hedges, L. V., Higgins, J. P. T., \& Rothstein, H. R. (2009). Introduction to Meta-Analysis. West Sussex, UK: Wiley.

Buist, A. E., Austin, M.-P. V., Hayes, B. A., Speelman, C., Bilszta, J. L. C., Gemmill, A. W., \& Milgrom, J. (2008). Postnatal Mental Health of Women Giving Birth in Australia 2002-2004: Findings from the beyondblue National Postnatal Depression Program. Australian and New Zealand Journal of Psychiatry, 42, 66-73. http://dx.doi.org/10.1080/00048670701732749

Cohen, J. (1988). Statistical Power Analysis for the Behavioral Sciences (2nd ed.). Hillsdale, NJ: Lawrence Erlbaum Associates.

Cotton, S. M., Wright, A., Harris, M. G., Jorm, A. F., \& McGorry, P. D. (2006). Influence of Gender on Mental Health Literacy in Young Australians. Australian and New Zealand Journal of Psychiatry, 40, 790-796. http://dx.doi.org/10.1111/j.1440-1614.2006.01885.x

Cuijpers, P., Brännmark, J. G., \& van Straten, A. (2008). Psychological Treatment of Postpartum Depression: A Meta-Analysis. Journal of Clinical Psychology, 64, 103-118. http://dx.doi.org/10.1002/jclp.20432

Department of Health and Ageing (2009). Framework for the National Perinatal Depression Initiative 2008-09 to 2012-13. http://www.health.gov.au/internet/main/publishing.nsf/Content/D617C5D13F84437BCA257BF0001ACD7D/\$File/perinat .pdf

Donahue Jennings, K., Ross, S., Popper, S., \& Elmore, M. (1999). Thoughts of Harming Infants in Depressed and Nondepressed Mothers. Journal of Affective Disorders, 54, 21-28. http://dx.doi.org/10.1016/S0165-0327(98)00185-2

Fairbrother, N., \& Woody, S. R. (2008). New Mothers' Thoughts of Harm Related to the Newborn. Archives of Women's Mental Health, 11, 221-229. http://dx.doi.org/10.1007/s00737-008-0016-7

Farrer, L., Leach, L., Griffiths, K., Christensen, H., \& Jorm, A. (2008). Age Differences in Mental Health Literacy. BMC Public Health, 8, 125. http://www.biomedcentral.com/1471-2458/8/125

Fernandez, R. J. (1992). Recent Clinical Management Experience. In J. A. Hamilton, \& P. N. Harberger (Eds.), Postpartum Psychiatric Illness: A Picture Puzzle (pp. 78-89). Philadelphia, PA: University of Pennsylvania Press.

Figueiredo, B., Costa, R., Pacheco, A., \& Pais, A. (2009). Mother-to-Infant Emotional Involvement at Birth. Maternal and Child Health Journal, 13, 539-549. http://dx.doi.org/10.1007/s10995-008-0312-x

Fisher, L. J., \& Goldney, R. D. (2003). Differences in Community Mental Health Literacy in Older and Younger Australians. International Journal of Geriatric Psychiatry, 18, 33-40. http://dx.doi.org/10.1002/gps.769

Forman, D. R., O’Hara, M. W., Stuart, S., Gorman, L. L., Larsen, K. E., \& Coy, K. C. (2007). Effective Treatment for Postpartum Depression Is Not Sufficient to Improve the Developing Mother-Child Relationship. Development and Psychopathology, 19, 585-602. http://dx.doi.org/10.1017/S0954579407070289

Goldney, R. D., Fisher, L. J., \& Wilson, D. H. (2001). Mental Health Literacy: An Impediment to the Optimum Treatment of Major Depression in the Community. Journal of Affective Disorders, 64, 277-284. http://dx.doi.org/10.1016/S0165-0327(00)00227-5

Goldney, R. D., Fisher, L. J., Wilson, D. H., \& Cheok, F. (2000). Major Depression and Its Associated Morbidity and Quality of Life in a Random, Representative Australian Community Sample. Australian and New Zealand Journal of Psychiatry, 34, 1022-1029. http://dx.doi.org/10.1046/j.1440-1614.2000.00836.x

Hendrick, V. (2003). Treatment of Postnatal Depression: Effective Interventions Are Available, but the Condition Remains Underdiagnosed. British Medical Journal, 327, 1003-1004. http://dx.doi.org/10.1136/bmj.327.7422.1003

Highet, N. J., Gemmill, A. W., \& Milgrom, J. (2011). Depression in the Perinatal Period: Awareness, Attitudes and Knowledge in the Australian Population. Australian and New Zealand Journal of Psychiatry, 45, 223-231.

http://dx.doi.org/10.3109/00048674.2010.547842 
Highet, N. J., Luscombe, G. M., Davenport, T. A., Burns, J. M., \& Hickie, I. B. (2006). Positive Relationships between Public Awareness Activity and Recognition of the Impacts of Depression in Australia. Australian and New Zealand Journal of Psychiatry, 40, 55-58. http://dx.doi.org/10.1080/j.1440-1614.2006.01742.x

Jorm, A. F., \& Kelly, C. M. (2007). Improving the Public’s Understanding and Response to Mental Disorders. Australian Psychologist, 42, 81-89. http://dx.doi.org/10.1080/00050060701280565

Jorm, A. F., Korten, A. E., Jacomb, P. A., Christensen, H., Rodgers, B., \& Pollitt, P. (1997). “Mental Health Literacy”: A Survey of the Public's Ability to Recognise Mental Disorders and Their Beliefs about the Effectiveness of Treatment. Medical Journal of Australia, 166, 182-186.

Letourneau, N., Duffett-Leger, L., Stewart, M., Hegadoren, K., Dennis, C. L., Rinaldi, C. M., \& Stoppard, J. (2007). Canadian Mothers' Perceived Support Needs during Postpartum Depression. Journal of Obstetric, Gynecologic, \& Neonatal Nursing, 36, 441-449. http://dx.doi.org/10.1111/j.1552-6909.2007.00174.x

Martinez, R., Johnston-Robledo, I., Ulsh, H. M., \& Chrisler, J. C. (2001). Singing “The Baby Blues”: A Content Analysis of Popular Press Articles about Postpartum Affective Disturbances. Women \& Health, 31, 37-56. http://dx.doi.org/10.1300/J013v31n02_02

Milgrom, J., Gemmill, A. W., Bilszta, J. L., Hayes, B., Barnett, B., Ericksen, J., Ellwood, D., \& Buist, A. (2008). Antenatal Risk Factors for Postnatal Depression: A Large Prospective Study. Journal of Affective Disorders, 108, 147-157. http://dx.doi.org/10.1016/j.jad.2007.10.014

Moehler, E., Brunner, R., Wiebel, A., Reck, C., \& Resch, F. (2006). Maternal Depressive Symptoms in the Postnatal Period Are Associated with Long-Term Impairment of Mother-Child Bonding. Archives of Women's Mental Health, 9, $273-278$. http://dx.doi.org/10.1007/s00737-006-0149-5

Murray, L., \& Cooper, P. J. (1999). Postpartum Depression and Child Development. New York: Guildford Press.

Robertson, E., Grace, S., Wallington, T., \& Stewart, D. (2004). Antenatal Risk Factors for Postpartum Depression: A Synthesis of Recent Literature. General Hospital Psychiatry, 26, 289-295.

http://dx.doi.org/10.1016/j.genhosppsych.2004.02.006

Schanie, C. L., Pinto-Foltz, M. D., \& Logsdon, M. C. (2008). Analysis of Popular Press Articles Concerning Postpartum Depression: 1998-2006. Issues in Mental Health Nursing, 29, 1200-1216. http://dx.doi.org/10.1080/01612840802370509

Thompson, A., Issakidis, C., \& Hunt, C. (2008). Delay to Seek Treatment for Anxiety and Mood Disorders in an Australian Clinical Sample. Behaviour Change, 25, 71-84. http://dx.doi.org/10.1375/bech.25.2.71

Turner, K. M., Sharp, D., Folkes, L., \& Chew-Graham, C. (2008). Women’s Views and Experiences of Antidepressants as a Treatment for Postnatal Depression: A Qualitative Study. Family Practice, 25, 450-455. http://dx.doi.org/10.1093/fampra/cmn056

Whiteford, H., Buckingham, B., \& Manderscheid, R. (2002). Australia’s National Mental Health Strategy. British Journal of Psychiatry, 180, 210-215. http://dx.doi.org/10.1192/bjp.180.3.210 
Scientific Research Publishing (SCIRP) is one of the largest Open Access journal publishers. It is currently publishing more than 200 open access, online, peer-reviewed journals covering a wide range of academic disciplines. SCIRP serves the worldwide academic communities and contributes to the progress and application of science with its publication.

Other selected journals from SCIRP are listed as below. Submit your manuscript to us via either submit@scirp.org or Online Submission Portal.
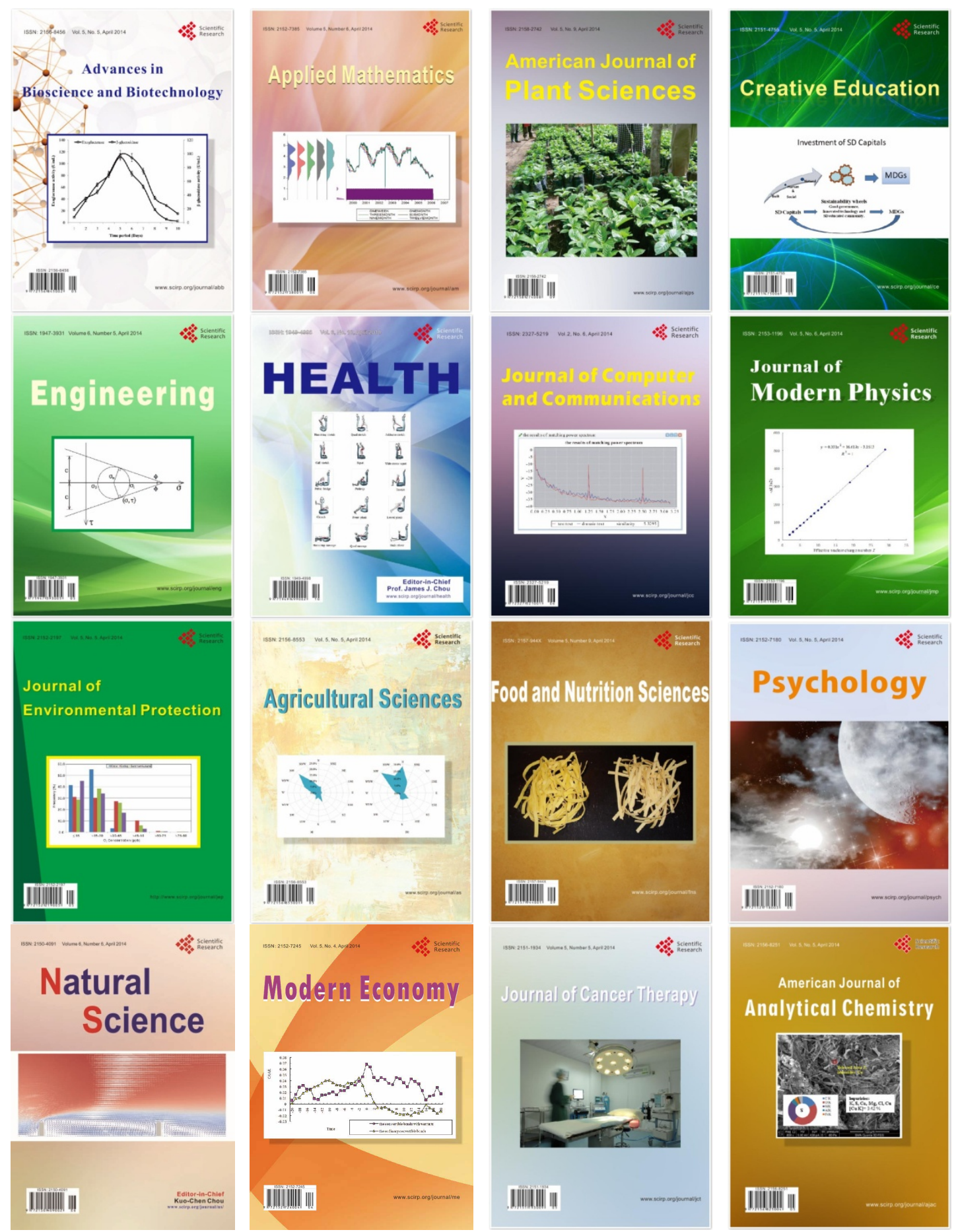